\title{
Studies of the diffractive photoproduction of isolated photons at HERA
}

\section{P. J. Bussey}

School of Physics and Astronomy

University of Glasgow

Glasgow, United Kingdom, G12 8QQ

E-mail: peter.bussey@glasgow.ac.uk

for the ZEUS Collaboration

The ZEUS detector at HERA has been used to measure the photoproduction of isolated photons in diffractive events. Cross sections are evaluated in the photon transverse-energy and pseudorapidity ranges $5<E_{T}^{\gamma}<15 \mathrm{GeV}$ and $-0.7<\eta^{\gamma}<0.9$, inclusively and with a jet with transverse energy and pseudorapidity in the ranges $4<E_{T}^{\text {jet }}<35 \mathrm{GeV}$ and $-1.5<\eta^{\text {jet }}<1.8$, for a total integrated luminosity of $456 \mathrm{pb}^{-1}$. Further kinematic variables studied include the fractions of the incoming photon energy and of the colourless exchange ("Pomeron") energy that are imparted to a photon-jet final state. Comparison is made to predictions from the RAPGAP Monte Carlo simulation.

XXV International Workshop on Deep-Inelastic Scattering and Related Subjects

3-7 April 2017

University of Birmingham, $U K$ 
Diffractive hadronic interactions involve the exchange of a colour-neutral object known as the "Pomeron". Diffractive scattering off protons may be initiated by a second incoming hadron, or by a real or virtual photon. At the HERA ep collider, diffractive processes have been studied both in photoproduction and in deep inelastic scattering, the photoproduction processes consisting of those in which the exchanged virtual photon is quasi-real. The diffractive process is characterised by a forward nucleon, followed by a "gap" in rapidity in which little or no energetic scattering is found until the central region of the process where the hard final state is detected and measured.

This talk presents measurements of diffractive photoproduction at HERA in which a hard isolated "prompt" photon is detected in the central region of the ZEUS detector and may be accompanied by a jet [1]. Such processes, while rare, are interesting for several reasons. An outgoing photon must be radiated from a charged partonic object, namely a quark, and therefore demonstrates the presence of a quark content in the Pomeron or of scattering in which both the Pomeron and incident photon couple to quarks. In general, they allow QCD-based models to be tested in order to improve our understanding of a type of process which is important at high energies.

It is possible for a photon to be radiated within a jet. Such processes are hard to model within QCD and can be suppressed by requiring that the outgoing photon be isolated. The present measurements follow an earlier study by $\mathrm{H} 1$ [2] of inclusive diffractive high energy prompt photons as a function of transverse momentum. Analyses of isolated photons in non-diffractive photoproduction have also been made by the ZEUS and H1 collaborations [3, 4].

\section{Kinematic quantities}

When a proton, with energy $E_{p}$, radiates a Pomeron or equivalent, the fraction of the proton energy taken by the radiated Pomeron is given to a good approximation by the variable $x_{\mathbb{P}}=$ $\left(E^{\text {all }}+p_{Z}^{\text {all }}\right) / 2 E_{p}$, where the suffix "all" refers to all final-state particles or detector-measured objects apart from the forward proton and its possible dissociation products. In "direct" photoproduction processes (fig. 1(a)), the entire energy of the incoming photon is absorbed by the target, while in "resolved" processes (fig. 1(b)), the incoming photon's hadronic structure provides a quark or gluon that interacts with a parton from the target. These two classes of process are unambiguously defined only at lowest order, but may be partially distinguished in events containing a high- $E_{T}$ photon and a jet by means of $x_{\gamma}^{\text {meas }}=\left(E^{\gamma}+E^{\text {jet }}-p_{Z}^{\gamma}-p_{Z}^{\text {jet }}\right) /\left(E^{\text {all }}-p_{Z}^{\text {all }}\right)$, which measures the fraction of the incoming photon energy that is given to the photon and the jet. The quantities $E^{\gamma}$ and $E^{\text {jet }}$ de-

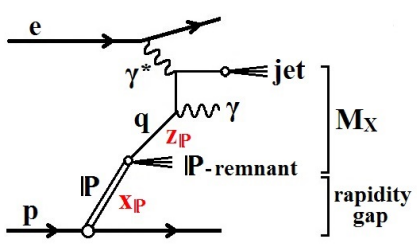

(a)

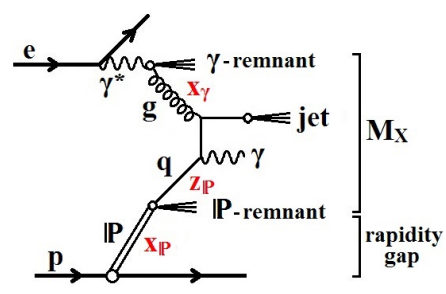

(b)

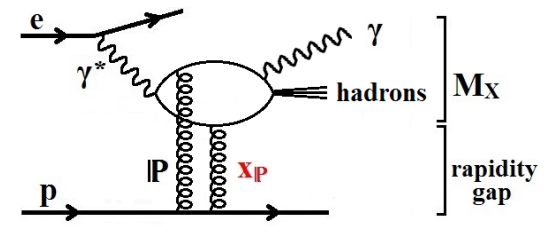

(c)

Figure 1: Examples of the diffractive production of a prompt photon and a jet in ep scattering of a resolved Pomeron from (a) direct (b) resolved photons, and (c) a direct Pomeron process. 
note the energies of the photon and the jet, respectively, $p_{Z}$ denotes the corresponding longitudinal momenta. The presence of direct processes generates a prominent peak in the cross section at high $x_{\gamma}^{\text {meas }}$ values. Similarly, direct and resolved Pomeron processes may be defined. The fraction of the Pomeron energy that is taken by the outgoing photon and jet is given to a good approximation by: $z_{\mathbb{P}}^{\text {meas }}=\left(E^{\gamma}+E^{\text {jet }}+p_{Z}^{\gamma}+p_{Z}^{\text {jet }}\right) /\left(E^{\text {all }}+p_{Z}^{\text {all }}\right)$. Figure 1(c) illustrates a possible event type in which the Pomeron is emulated by two exchanged gluons and there is no Pomeron remnant.

\section{Experimental method}

The measurements are based on two data samples corresponding to integrated luminosities of 82 and $374 \mathrm{pb}^{-1}$, taken during the years 1998-2000 and 2004-2007 respectively with the ZEUS detector at HERA, and referred to as HERA-I and HERA-II data respectively. During these periods, HERA ran with an electron or positron beam energy of $27.5 \mathrm{GeV}$ and a proton beam energy of $E_{p}=920 \mathrm{GeV}$. The samples include $e^{+} p$ and $e^{-} p$ data $^{1}$.

Charged particles were measured in the ZEUS central tracking detector and a silicon micro vertex detector, which operated in a solenoidal field of 1.43 T. ZEUS used a uranium-scintillator calorimeter, of which the barrel electromagnetic calorimeter (BEMC) had cells with a pointing geometry aimed at the nominal interaction point. Its fine granularity allowed the use of showershape distributions in the measurement of outgoing high-energy photons.

Monte Carlo (MC) event samples were employed to evaluate the detector acceptance and event-reconstruction efficiency, and to provide signal and background distributions. RAPGAP 3.2 [5] was used to generate the diffractive process $p e \rightarrow p e \gamma X$ for direct and resolved incoming virtual photons at low $Q^{2}$, incident on resolved Pomerons modelled according to the approach of Ingelman and Schlein. The diffractive proton PDF set H1 DPDF Fit B (2006) was used and, for the resolved photon, the PDF set SASGAM 2D. The program PYTHIA 6.416 was used to generate direct and resolved prompt-photon photoproduction processes for background calculations.

The measured photons are accompanied by backgrounds from neutral mesons in hadronic jets, in particular $\pi^{0}$ and $\eta$, where the meson decay products can create an energy cluster in the BCAL that passes the selection criteria for a photon. These were modelled using RAPGAP events with a dijet final state in which one of the jets resembled a photon candidate. Further backgrounds came from DIS events and Bethe-Heitler events that produced a photon-electron final state. These were simulated using DJANGOH 6 interfaced with HERAClES, and with GRAPE-COMPTON.

The basic event selection and reconstruction were performed as previously [3]. A three-level trigger system was used to select events online. The event analysis made use of energy-flow objects (EFO's), constructed from clusters of calorimeter cells, associated with tracks when appropriate, and also unassociated tracks. Photon candidates were identified as EFO's with no associated track, and with at least $90 \%$ of the reconstructed energy measured in the BEMC.

Jets were reconstructed using all the EFO's in the event, including photon candidates, by means of the $k_{T}$ clustering algorithm in the $E$-scheme in the longitudinally invariant inclusive mode with the radius parameter set to 1.0. To reduce the fragmentation contribution and the background from the decay of neutral mesons within jets, the photon candidate was required to be isolated from other

\footnotetext{
${ }^{1}$ Hereafter "electron" refers to both electrons and positrons unless otherwise stated.
} 
hadronic activity. This was imposed by requiring that the photon-candidate EFO had at least $90 \%$ of the total energy of the reconstructed jet of which it formed a part.

Events were finally selected with the following kinematic conditions. Each event was required to contain a photon candidate with a reconstructed transverse energy, $E_{T}^{\gamma}$, in the range $5<E_{T}^{\gamma}<15 \mathrm{GeV}$ and with pseudorapidity, $\eta^{\gamma}$, in the range $-0.7<\eta^{\gamma}<0.9$. The hadronic jet, when used, was required to have $E_{T}^{\text {jet }}$ between 4 and $35 \mathrm{GeV}$ and to lie within the pseudorapidity, $\eta^{\text {jet }}$, range $-1.5<\eta^{\text {jet }}<1.8$. To select diffractive events further conditions were applied, the first of which was that the maximum pseudorapdity for EFO's with energy above $0.4 \mathrm{GeV}$ satisfied $\eta_{\max }<2.5$. A second diffractive condition was that $x_{\mathbb{P}}<0.03$. For the HERA-I data sample, the energy in the Forward Plug Calorimeter was required to be less than $1 \mathrm{GeV}$. This calorimeter was not present in the HERA-II running. A serious background consisted of Bethe-Heitler events containing a high- $E_{T}$ photon and electron in the final state. These and DIS events were efficiently removed by rejecting events with an identified electron and less than six EFO's in the detector.

The results are potentially affected by proton-dissociation processes, in which the products of the proton dissociation pass undetected inside the central aperture of the Forward Calorimeter. These were not modelled in the version of RAPGAP that was used. In other ZEUS diffracrive analyses, these were estimated to be up to $40 \%$ for the HERA-II data and up to $16 \%$ for the HERAI data. The HERA-II data sample was used in the main analysis described here to give the best estimation of the shapes of the cross-section distributions, which were assumed not to depend on the presence of proton dissociation. The HERA-I data were analysed similarly, with the addition of the selection on the Forward Plug Calorimeter, and were used to evaluate the total cross section within the selected parameter range.

Background events arising from neutral meson decays were subtracted statistically, following the approach taken in previous ZEUS analyses. Studies of the event shapes using PYTHIA indicated that the fraction of non-diffractive events in the sample lies between $0 \%$ and $10 \%$. This was included in the systematic uncertainties, which were otherwise dominated by the uncertainties in the calorimeter and jet calibration.

\section{Results}

Differential cross sections were calculated for the diffractive production of an isolated photon, inclusive and with at least one accompanying jet. The kinematic region was defined by $Q^{2}<$ $1 \mathrm{GeV}^{2}, 0.2<y<0.7,-0.7<\eta^{\gamma}<0.9,5<E_{T}^{\gamma}<15 \mathrm{GeV}, 4<E_{T}^{\text {jet }}<35 \mathrm{GeV}$ and $-1.5<\eta^{\text {jet }}<$ 1.8. The diffractive condition consisted in requirements that $x_{\mathbb{P}}<0.03$ and $\eta^{\max }<2.5$. These quantities were evaluated at the hadron level in the laboratory frame, matching the cuts used at the detector level, and the jets were formed according to the $k_{T}$ clustering algorithm with the radius parameter set to 1.0. Photon isolation was imposed such that at least $90 \%$ of the energy of the jet-like object containing the photon originated from the photon.

The results here are inclusive of proton dissociation processes which have been estimated to comprise approximately $16 \%$ of the total diffractive cross section. The total cross section within the observed parameter ranges was found to be $0.68 \pm 0.14_{-0.07}^{+0.06} \mathrm{pb}$, to be compared to the RAPGAP prediction of $0.68 \mathrm{pb}$ with no proton dissociation and no resolved-suppression factor. It is found 
(a)

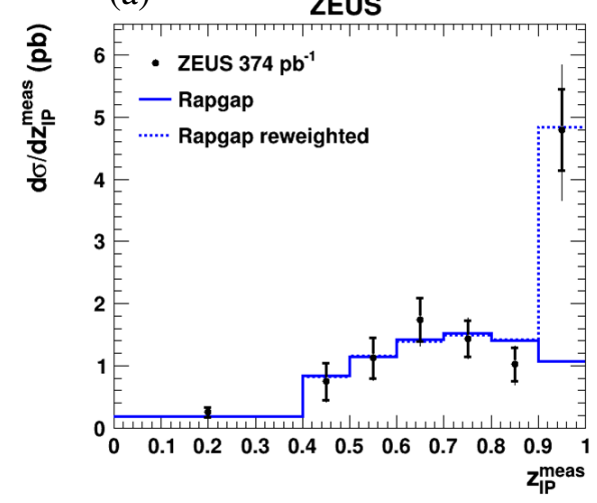

(b)

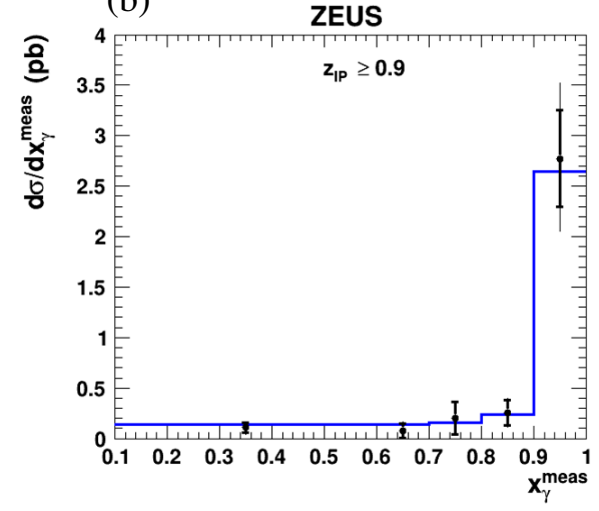

Figure 2: Differential cross sections for events containing an isolated photon accompanied by a jet: (a) as a function of $z_{\mathbb{P}}^{\text {meas }}$; (b) as a function of $x_{\gamma}^{\text {meas }}$ for events with $z_{\mathbb{P}}^{\text {meas }}>0.9$ at the hadron level. Comparisons are made to normalised predictions from RAPGAP, with and without reweighting. Thick error bars are statistical and thin are statistical combined with systematic.

that a high fraction of the events with an observed isolated high- $E_{T}$ photon are accompanied by at least one jet.

Figure 2(a) shows the differential cross section for $z_{\mathbb{P}}^{\text {meas }}$. At the upper end of the distribution, a peak is seen which is not described by RAPGAP and which gives evidence for the presence of directPomeron processes. The solid RAPGAP histogram is normalised to the region $z_{\mathbb{P}}^{\text {meas }}<0.9$, while the dashed histogram includes a reweighting factor and is normalised to the entire data distribution. Figure 2(b) shows the $x_{\gamma}^{\text {meas }}$ cross section for events with $z_{\mathbb{P}}^{\text {meas }}>0.9$ and shows that these events are dominated by a direct-photon component. Figure 3 shows cross sections for a selection of other variables, for the whole data set. The RAPGAP histograms are normalised to the data and describe the shapes of the measured quantities well. Cross sections in $E_{T}^{\mathrm{jet}}$ above $15 \mathrm{GeV}$ are omitted from fig. 3(c) owing to limited statistics, but this kinematic region is included in the other cross-section measurements. Figure 3(e) shows the difference in azimuth between the photon and the jet and demonstrates the back-to-back nature of these events. Figure 3(f) shows that the $x_{\mathbb{P}}$ distribution is well-contained within the selection criterion that was applied.

\section{References}

[1] ZEUS Collaboration, H. Abramowicz et al., arXiv:1705.10251

[2] H1 Collaboration, V. Andreev et al., Phys. Lett. B 672 (2009) 219

[3] ZEUS Collaboration, H. Abramowicz et al., Phys. Lett. B 730 (2014) 293

[4] ZEUS Collaboration, J. Breitweg et al., Phys. Lett. B 413 (1997) 201 ZEUS Collaboration, J. Breitweg et al., Phys. Lett. B 472 (2000) 175 ZEUS Collaboration, S. Chekanov et al., Phys. Lett. B 511 (2001) 19 ZEUS Collaboration, S. Chekanov et al., Eur. Phys. J. C 49 (2007) 511 H1 Collaboration, A. Aktas et al., Eur. Phys. J. C 38 (2004) 437

[5] H. Jung, http://www.desy.de/ jung/rapgap.html 
(a)

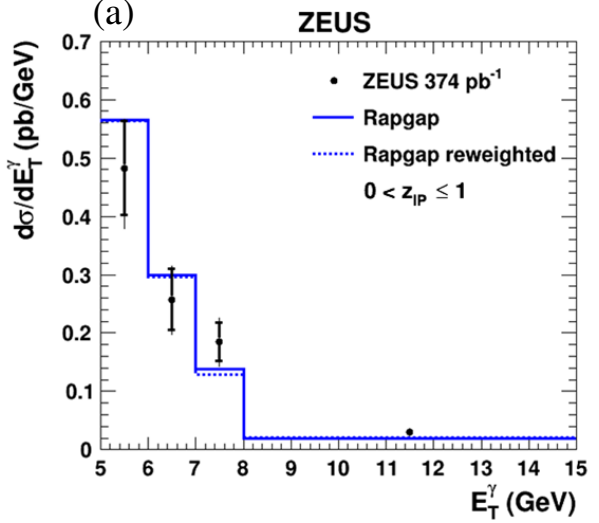

(c)

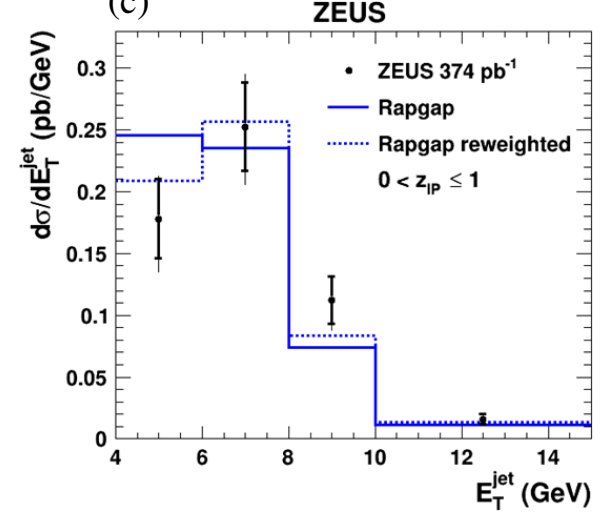

(e)

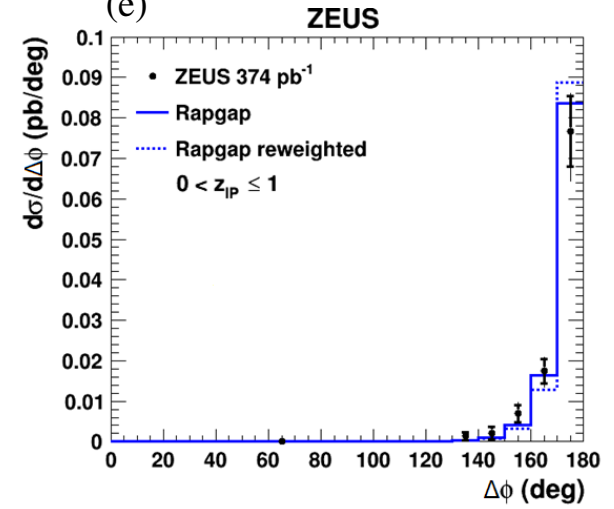

(b)

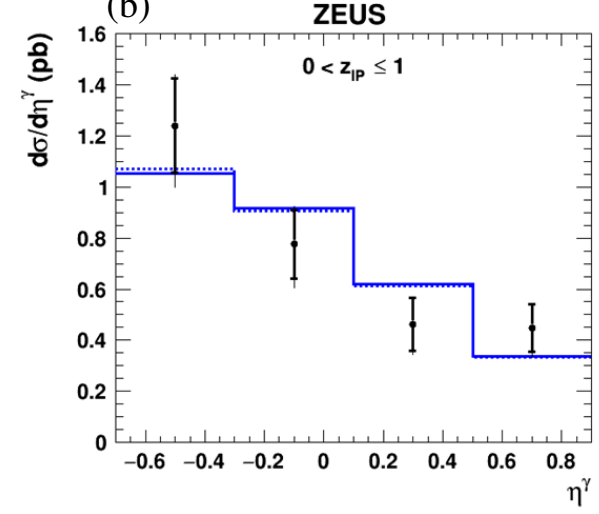

(d)

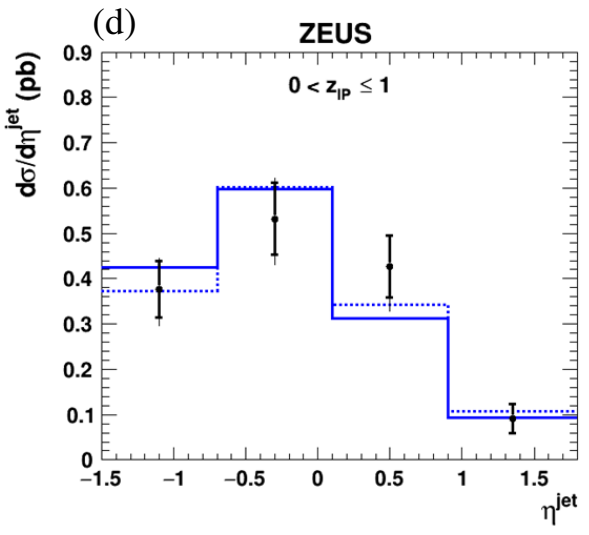

(f)

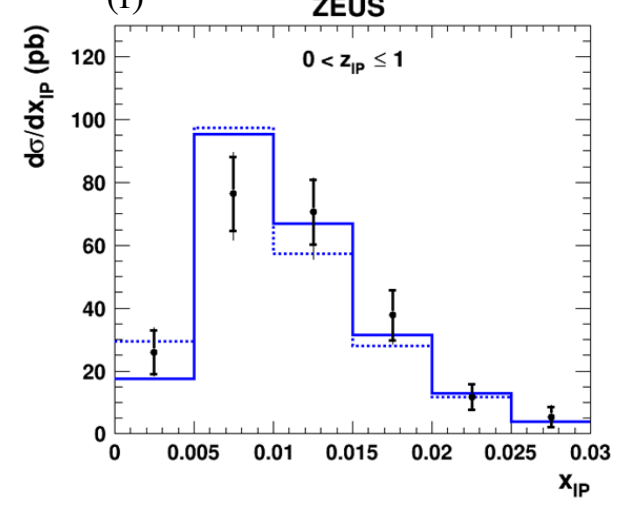

Figure 3: Differential cross sections for events containing an isolated photon accompanied by a jet as functions of: (a) $E_{T}^{\gamma}$, (b) $\eta^{\gamma}$, (c) $E_{T}^{\text {jet }}$, (d) $\eta^{\text {jet }}$, (e) $\Delta \phi$, and (f) $x_{\mathbb{P}}$. 